\title{
Evaluation of Averted Doses to Infants by Tap Water Restrictions after the Fukushima Daiichi Nuclear Power Plant Accident
}

\author{
Sakae KINASE ${ }^{1, *}$, Masanori KIMURA ${ }^{1}$, Shogo TAKAHARA ${ }^{1}$ \\ and Toshimitsu HOMMA ${ }^{1}$ \\ ${ }^{1}$ Nuclear Safety Research Center, Japan Atomic Energy Agency, 2-4 Shirane, Shirakata, Tokai-mura, \\ Naka-gun, Ibaraki 319-1195, Japan
}

\begin{abstract}
There has been some concern in reviewing the effectiveness of making decisions on the implementation of protective measures in emergency exposure situations. After the Fukushima Daiichi Nuclear Power Plant accident, temporal changes in the concentration of iodine 131 in tap water were studied using published data from several authorities in Fukushima, Ibaraki, and Tokyo. Averted doses to infants (1-year-old children) due to the intake of iodine 131 through tap water intake restrictions were also evaluated. Consequently, it was found that the apparent half-life of iodine 131 in tap water was 2.8 days. The averted equivalent doses to the thyroids of 1-year-old children were found to have a maximum value of $8.3 \mathrm{mSv}$ in a local area of Fukushima. Hence, the tap water intake restrictions implemented by the authorities were considered to be effective in the early phase of the emergency exposure situation.
\end{abstract}

KEYWORDS: Fukushima, nuclear accident, iodine, tap water, infant, thyroid, dose

\section{Introduction}

In response to the Fukushima Daiichi Nuclear Power Plant Accident that occurred on March 11,2011 , tap water intake restrictions were implemented based on radioactive iodine 131 (hereinafter ${ }^{131} \mathrm{I}$ ) in Fukushima and in a wide range of areas including Ibaraki and Tokyo. However, there was no specific approach for canceling the tap water intake restrictions, which resulted in social confusion because the decision for the cancellation determination was left to the local governments that operated the water supply. Considering this situation, it is essential to evaluate the averted doses through the tap water intake restrictions, i.e., the effectiveness of preventive measures, and to conduct a survey on the characteristics of variations in ${ }^{131}$ I concentration in tap water. In this study, to contribute to the study on the establishment of standards for the cancellation of future tap water intake restrictions, the averted doses to infants (1-year-old children) through the tap water intake restrictions were evaluated. A survey on the variations in ${ }^{131}$ I concentration was also conducted in the tap water of Fukushima, Ibaraki and Tokyo, all of

\footnotetext{
${ }^{*}$ Corresponding author, E-mail: kinase.sakae@jaea.go.jp

DOI : 10.15669/fukushimainsights. Vol.4.6

(C) 2021 Atomic Energy Society of Japan. All rights reserved.

Originally published in Transactions of the Atomic Energy Society of Japan (ISSN 1347-2879), Vol. 10, No. 3, p.149-151

(2011) in Japanese. (Japanese version accepted: June 14, 2011)
} 
which were directly influenced by the accident at the Fukushima Daiichi Nuclear Power Plant.

\section{Actions Implemented by the Ministry of Health, Labour and Welfare (MHLW) on Tap Water Intake by Infants}

On March 19, 2011, the MHLW declared an index value (radioactive iodine concentration: $300 \mathrm{~Bq} / \mathrm{kg}$ ), which was determined by the Nuclear Safety Commission. Furthermore, on March 21,2011 , in order for the temporary restriction value to accord with the food sanitation law, it was recommended that the intake of the tap water, such as through the feeding of modified milk powder dissolved in tap water, should be avoided in infants when the radioactive iodine concentration exceeded $100 \mathrm{~Bq} / \mathrm{kg}$. On April 4, 2011, the time at which the tap water intake restrictions should be cancelled was defined as when the average of radioactive iodine concentration in the tap water, over a period of three days, falls below the prescribed standard and continues on a decreasing trend.

\section{Actions Implemented by the Local Governments on Tap Water Intake by Infants}

The local governments in Fukushima, Ibaraki and Tokyo implemented restrictions on tap water intake by infants in accordance with the standard established by the Nuclear Safety Commission or the MHLW, and then cancelled this preventative measure at their own discretion (Table 1). The restriction on tap water intake by infants in Tokyo was triggered by the $210 \mathrm{~Bq} / \mathrm{kg}$ detected in the sample taken at the Kanamachi Purification Plant on March 22, 2011.

Table 1 Tap water intake restrictions implemented by the authorities

\begin{tabular}{ccccc}
\hline Prefectures & Authority & The first day & The last day & Days \\
\hline Fukushima & Iitate & March 21st & May 10th & 50 \\
& $\begin{array}{c}\text { Koriyama, } \\
\text { Kawamata } \\
\text { Minamisoma }\end{array}$ & March 22nd & March 25th & 3 \\
& Iwaki & March 22nd & March 30th & 8 \\
& Tokai & March 23rd & March 31st & 8 \\
Ibaraki & The 23 wards and 5 cities & March 23rd & March 24th & 3 \\
Tokyo & &
\end{tabular}

\section{Methodology}

Using the data published by the MHLW, the Tokai Village Office and the Tokyo Metropolitan Office ${ }^{1)}$, a survey was conducted on the variations in ${ }^{131}$ I concentration in the tap water sampled from five local governments in Fukushima (Takishita Purification Plant, Iitate Village; Toyota-Machi-Jinai, Koriyama City; Aza Toinokuchi-Jinai, Kawamata Town; Minamisoma City government office complex; and Iwaki City government office complex); the Tojuku Purification Plant, Tokai Village, Ibaraki Prefecture; and Hyakunincho, Shinjuku, Tokyo. The time period for the survey was one month from March 16 to April 16, 2011 (till May 10, 2011 in Iitate Village). Furthermore, the amount of daily ${ }^{131} \mathrm{I}$ intake by 1 -year-old infants was estimated 
based on the ${ }^{131} I$ concentration in the tap water and the quantity of daily water intake. The equivalent infant thyroid dose rate and the effective dose rate were estimated by multiplying the ${ }^{131}$ I intake with the dose coefficient from the International Commission on Radiological Protection (ICRP) Publication 67 ${ }^{2}$. Referring to the values indicated in the Safety Series No. $81^{\text {3) }}$ of the International Atomic Energy Agency (IAEA), the volume of water intake of a 1-year-old infant was assumed to be $1.4 \times 10^{3} \mathrm{~mL}$ per day. Table 2 shows the dose coefficient of ${ }^{131} \mathrm{I}$. Note that for days on which sampling was not conducted, dose evaluation was conducted using the value obtained for a sample measured on the day before or the day after, whichever value was the higher. For days on which ${ }^{131} \mathrm{I}$ was not detected in the tap water, it was assumed that no significant exposure had occurred.

\begin{tabular}{|c|c|c|}
\hline Arc of intakes & Thyroid equivalent dose & Effective dose \\
\hline 1-year-old children & $3.6 \times 10^{-6}$ & $1.8 \times 10^{-7}$ \\
\hline Adult ${ }^{a)}$ & $4.3 \times 10^{-7}$ & $2.2 \times 10^{-8}$ \\
\hline
\end{tabular}

\section{Results and Discussions}

Figure 1 presents the variations in ${ }^{131} \mathrm{I}$ concentration in the tap water observed in the five local governments in Fukushima Prefecture (Iitate Village, Koriyama City, Kawamata Town, Minamisoma City, and Iwaki City), Tokai Village in Ibaraki and Shinjuku in Tokyo. As shown in the chart, ${ }^{131}$ I concentration in the tap water varied according to the sampling location, and on March 26, 2011, 37.2 Bq/kg was observed as the maximum for Shinjuku, which is 230 $\mathrm{km}$ from the Fukushima Daiichi Nuclear Power Plant. ${ }^{131}$ I concentration in the tap water from Tokyo was lower compared to that of a number of locations in Fukushima Prefecture and Tokai

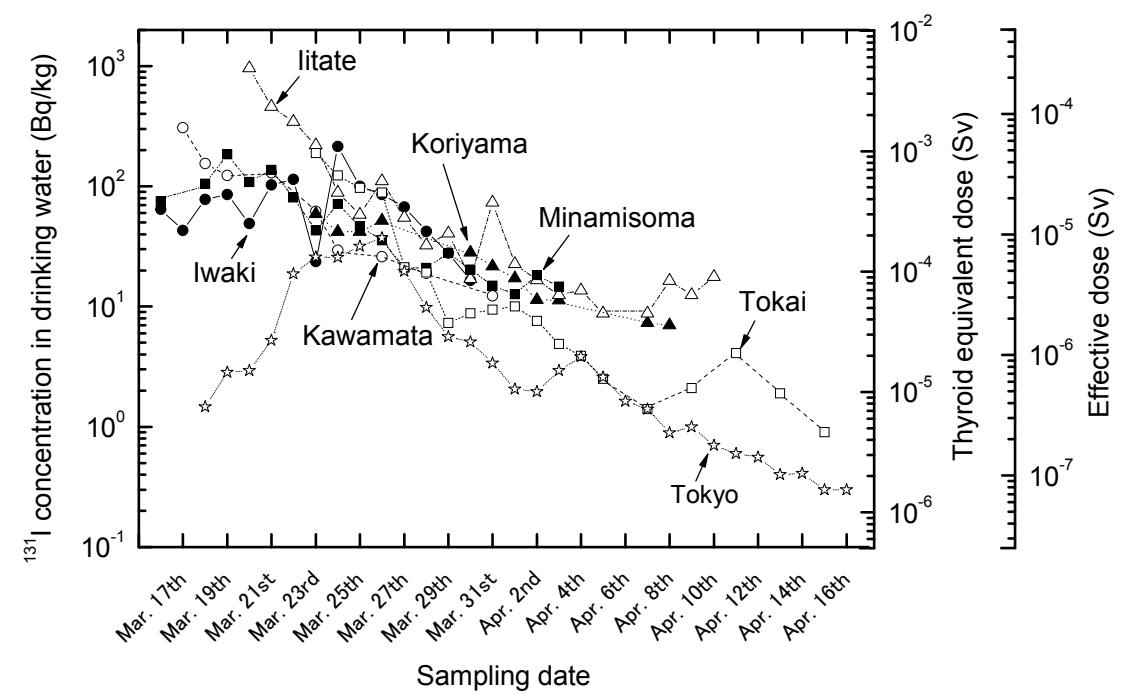

Figure 1 Concentration of ${ }^{131} \mathrm{I}$ in tap water, projected equivalent doses to thyroid, and effective doses of 1-year-old children, due to the intake of ${ }^{131} \mathrm{I}$ in tap water in local areas of Fukushima, Tokai and Tokyo 
Village in Ibaraki. Accordingly, it was confirmed that there had been no immediate influence traceable to the accident of Fukushima Daiichi Nuclear Power Plant. The general trend showed a decrease over time after the occurrence of the Fukushima Daiichi Accident in all locations. The reduction rate in the variations of ${ }^{131}$ I concentration in tap water displayed an apparent half-life period of approximately $2.8^{+/}-1.2$ days, which is shorter than the physical half-life of ${ }^{131} \mathrm{I}$ of 8.0 days. This trend was observed due to the reduced additional precipitation of ${ }^{131} \mathrm{I}$ in the relevant locations after March 16, 2011 when the survey began, and accordingly, the dilution effect in the environment was considered to have contributed significantly to these findings. After April 1, 2011, a low concentration of ${ }^{131}$ I was detected in tap water from Iitate Village, Koriyama City, and Minamasoma City in Fukushima, Tokai Village in Ibaraki, and Shinjuku in Tokyo. It is assumed that these results are due to the inflow of deposits and re-floating of matter with the rain water into the tap water source. During the survey period, the highest value was detected in Iitate Village $(965 \mathrm{~Bq} / \mathrm{kg})$.

Additionally, Figure 1 shows the variations in the thyroid equivalent dose and the effective dose rate for an infant. All dose rates demonstrated a decreasing trend over time along with the variations in ${ }^{131} \mathrm{I}$ concentration in the tap water. Note that in the case of long-term, continuous, and chronic intake of ${ }^{131} \mathrm{I}$ by an infant, it is assumed that ${ }^{131} \mathrm{I}$ in the thyroid or inside the body builds up and then reaches the equilibrium value (= (effective half-life (days) $) \times($ constant intake amount per day) / $\ln 2$ ) due to the balancing of intake and egestion. Consequently, the thyroid equivalent dose and the effective dose rate are expected to be lower than the total sum of the daily evaluated dose rate.

Table 3 lists the averted dose in infants resulting from the tap water intake restrictions implemented by local governments. During the continued emergency exposure conditions, the tap water intake restrictions were an effective measure for avoiding a thyroid equivalent dose of $8.3 \mathrm{mSv}$ and effective dose rate of $4.1 \times 10^{-1} \mathrm{mSv}$ and for reducing the residual dose rate, especially in Iitate Village, Fukushima Prefecture.

Given the risk of exposure under the circumstances that prevailed at the time, not only to ${ }^{131}$ I but also to radioactive cesium $\left({ }^{137} \mathrm{Cs}\right)$ and other radioactive nuclides, the tap water intake restrictions implemented by the MHLW can be justified as a protective measure for decreasing the thyroid equivalent dose and the effective dose rate in infants. Furthermore, in areas such as Tokai Village, the supply of tap water was delayed due to the power outage caused by the earthquake and the works undertaken to restore the tap water pipelines. This delay in tap water supply happened to reduce the exposure to ${ }^{131} \mathrm{I}$ in the tap water. In comparison, in Shinjuku, Tokyo, where the duration of the tap water intake restriction was shorter and the concentration of ${ }^{131} \mathrm{I}$ was low, the averted thyroid equivalent dose in infants was $1.3 \times 10^{-1} \mathrm{mSv}$.

Table 3 Averted doses in 1-year-old children due to the intake of ${ }^{131} \mathrm{I}$ by tap water restrictions (Sv)

\begin{tabular}{ccc}
\hline Authority & Thyroid equivalent dose & Effective dose \\
\hline Iitate, Fukushima & $8.3 \times 10^{-3}$ & $4.1 \times 10^{-4}$ \\
Koriyama, Fukushima & $5.1 \times 10^{-4}$ & $2.5 \times 10^{-5}$ \\
Kawamata, Fukushima & $1.1 \times 10^{-3}$ & $5.6 \times 10^{-5}$ \\
Minamisoma, Fukushima & $1.7 \times 10^{-3}$ & $8.7 \times 10^{-5}$ \\
Iwaki, Fukushima & $2.9 \times 10^{-3}$ & $1.5 \times 10^{-4}$ \\
Tokai, Ibaraki & $2.1 \times 10^{-3}$ & $1.0 \times 10^{-4}$ \\
Shinjuku, Tokyo & $1.3 \times 10^{-4}$ & $6.5 \times 10^{-6}$ \\
\hline
\end{tabular}




\section{Conclusions}

A survey was conducted on variations in ${ }^{131}$ I concentration in the tap water of Fukushima Prefecture, Ibaraki Prefecture and Tokyo after the Fukushima Daiichi Nuclear Power Plant Accident. In addition to evaluating the thyroid equivalent dose to infants and the effective dose rate of ${ }^{131} \mathrm{I}$ in the tap water, the averted dose rate resulting from the intake restrictions implemented by the local governments was calculated. As a result, it became clear that the ${ }^{131} \mathrm{I}$ concentration in tap water was influenced heavily by the sample locations, and it decreased with the rate of the apparent half-life of approximately 2.8 days. The tap water intake restriction related to the Fukushima Daiichi Nuclear Power Plant was effective in reducing the thyroid equivalent dose and the averted thyroid equivalent dose to infants was approximately $8.3 \mathrm{mSv}$ in Iitate Village, Fukushima Prefecture.

\section{References}

1) Ministry of Health, Labour and Welfare, Information about the Great East Japan Earthquake, Online, Available: http://www.mhlw.go.jp/english/topics/2011eq/index.html (2011, April 17).

2) ICRP, Age-dependent Doses to Members of the Public from Intake of Radionuclides: Part 2 Ingestion Dose Coefficients, ICRP Publication 67, Pergamon Press, Oxford (1993).

3) IAEA, Derived Intervention Levels for Application in Controlling Radiation Doses to the Public in the Event of a Nuclear Accident or Radiological Emergency, IAEA, Vienna, Safety Series No. 81, IAEA, Vienna (1986). 